\title{
Development and Characterization of Soy Lecithin Liposome as Potential Drug Carrier Systems for Codelivery of Letrozole and Paclitaxel
}

\author{
Minh Thanh Vu $\mathbb{D}^{1,}{ }^{1,2}$ Ngoc Thuy Trang Le $\mathbb{D}^{2,3}{ }^{2,3}$ Truc Le-Buu Pham, ${ }^{4}$ Ngoc Hoi Nguyen $\mathbb{D}^{2,3}$ \\ and Dai Hai Nguyen $\mathbb{D}^{2,3}$ \\ ${ }^{1}$ Institute of Chemistry and Materials, 17 Hoang Sam, Cau Giay, Hanoi City 100000, Vietnam \\ ${ }^{2}$ Graduate University of Science and Technology, Vietnam Academy of Science and Technology, Hanoi City 100000, Vietnam \\ ${ }^{3}$ Institute of Applied Materials Science, Vietnam Academy of Science and Technology, Ho Chi Minh City 700000, Vietnam \\ ${ }^{4}$ Biotechnology Center of Ho Chi Minh City, 2374, Highway 1, Trung My Tay Ward, District 12, Ho Chi Minh City 700000, Vietnam
}

Correspondence should be addressed to Dai Hai Nguyen; nguyendaihai@iams.vast.vn

Received 19 August 2020; Revised 5 November 2020; Accepted 15 December 2020; Published 30 December 2020

Academic Editor: Garima Agrawal

Copyright $\odot 2020$ Minh Thanh Vu et al. This is an open access article distributed under the Creative Commons Attribution License, which permits unrestricted use, distribution, and reproduction in any medium, provided the original work is properly cited.

\begin{abstract}
In the present work, a dual-drug-loaded soy lecithin liposomal system was developed by coencapsulation of Letrozole (LET) with Paclitaxel (PTX) to improve the efficacy in breast cancer therapy. Liposomes were synthesized by the thin film layer hydration. To sufficiently evaluate the characteristics of these liposomes, the particle size, zeta potential, morphology, drug encapsulation, in vitro drug release, and cytotoxicity were ascertained. Results showed promisingly anticancer potentials, as the following parameters indicated: nanosize diameter (around $193 \mathrm{~nm}$ ) and negative surface charge. Data collected from the coloaded drug liposomes showed suitable encapsulation efficiency (50.56\% for PTX and 31.13\% for LET). Controlled and sustained releases were achieved up to $72 \mathrm{~h}$ for both the loaded drugs following the diffusion mechanism. In addition, the in vitro cytotoxicity study on the human breast cancer cell line (MCF-7) given the dual-drug-loaded liposome showed greater inhibition of cell growth than the single drug. Consequently, LET and PTX coloaded liposomes made from soy lecithin are expected to be an ingenious drugdelivery system for combination chemotherapy.
\end{abstract}

\section{Introduction}

Breast cancer therapy using oestrogen-targeted drugs is one of the most successful anticancer strategies to date. Accordingly, Letrozole (LET), a third-generation aromatase inhibitor, is the antioestrogen drug that is commonly used in the treatment of breast cancer $[1,2]$. By successfully preventing aromatase which produces oestrogen, LET slows down the growth of hormone-responsive breast tumours in vivo. However, research has shown that LET has some negative effects on patients, including diarrhoea, constipation, fever, fatigue, and chest pains [3]. Regarding its overall effects on human health, LET was reported to possess potential efficacy that could be enhanced by advancing in combination with other chemotherapeutic agents [4-7]. Therefore, researchers have currently focused on LET combination therapies, particularly with chemotherapy, to take advantage of therapies using multiple drugs including coordinately distributed drugs to specific sites, reduce toxicity, and increase efficacy of drugs, as well as slow the rate of developing drug resistance, in order to advance in the full potentials of chemodrug treatments [8]. In the process of theoretical searching for the chemotherapeutic agent in the LET combination therapy, Paclitaxel (PTX) seems to be a great candidate [9]. By preventing the cells from dividing and replicating and causing the death of the cells in various proposed mechanisms, PTX in combination with LET is expected to destroy any of the remaining cancer cells, which are left uninhibited during the developing of LET [9-11]. Chen et al. have published their study in AntiCancer Drugs, reporting that exemestane, one of the aromatase inhibitors (AIs), could combine with Paclitaxel for the treatment of aromatase-positive gynecological cancer [9]. 
This combination allowed reducing the Paclitaxel dosage and therefore the toxicity of the treatment. Besides, clinical trials for AIs, a class of drugs used in the treatment of breast cancer in postmenopausal women and gynecomastia in men, are underway. As a result of this, it is valuable and has a contributing meaning to the scientific community to develop a codelivery system utilizing LET and PTX as loaded drugs and to evaluate its efficacy in the current status of breast cancer treatment.

In the combination therapy of agents with different solubility and pharmacokinetic properties such as LET plus PTX, it is considered that finding the ideal carrier systems/materials is the biggest challenge. Accompanied by these difficulties, nanoparticles with the abilities to sufficiently encapsulate and deliver dissimilar anticancer agents have been developed. Notably, liposome-based nanoparticles have been rising as a part of the technologies that health organizations already adopted for treatment purposes because of numerous benefits [12-15]. Liposomes (Lips) are microscopic lamellar structures that can be formed in the admixture of the soy lecithin and cholesterol and completed by the subsequent hydration in aqueous media [16-18]. Regarding this, liposomes have been widely evaluated and utilized in the controlled as well as the targeted drug delivery as novel systems in cancer treatment. For instance, these systems enhance the delivery of drugs to specific body organs and cells; in some cases, they can even reduce drug toxicity and facilitate the administration of different kinds of diagnoses [19]. He and Ma found that these nanoparticles reduced drug dosage in the case of medication errors [1]. Moreover, liposomes were reported to successfully codeliver drugs in the combination of LET in therapy as well as PTX combination therapy with other agents [5, 20-22]. Therefore, liposomes are predicted to exert their promising properties in improving the therapeutic efficacy of LET by coloading and codelivery with PTX for the treatment of breast cancer cells with faster absorption of the medication into the body, reducing side effects, and enhanced maximal dose tolerance.

In this regard, the aim of this study was to develop soy lecithin liposomal systems for the coencapsulated LET and PTX. It should be noted that the proposed liposomes were made from soy lecithin and cholesterol, which were prepared by hydrating a thin lipid film then reducing the particle size distribution by sonication followed by extrusion. This study also assessed the properties of the named nanoparticles, including particle size, polydispersity index, zeta potential, and morphology. In addition, in vitro tests that entailed the drug loading and releasing efficiency were conducted. The cytotoxic effect of single and combined drugs in human breast cancer cell lines (MCF-7) was evaluated by WST assay. In summary, this study is aimed at the drug delivery system's preparation, investigation, and understanding of the application of LET in combination with PTX.

\section{Materials and Methods}

2.1. Materials. Letrozole (LET) was synthesized in the previous study [23]. Paclitaxel (PTX) was purchased from Samyang Corporation (Seoul, Korea). Soy lecithin and
Tween 80 (polyoxyethylene sorbitan monooleate) were obtained from Tokyo Chemical Industry Co., Ltd. (Tokyo, Japan). Cholesterol was supplied by Sigma-Aldrich (St Louis, MO, USA). Cetyltrimethylammonium bromide (CTAB) and all solvents were of analytical grade and obtained from Merck (Darmstadt, Germany). A dialysis bag (Spectra/Por, regenerated cellulose) was purchased from Spectrum Laboratories Inc. (Canada). The Dulbecco's Modified Eagle's Medium: Nutrient Mixture F-12 (DMEM/F-12) and fetal bovine serum (FBS) were purchased from Thermo Fisher Scientific (Ho Chi Minh City, Vietnam). Human breast cancer cell lines (MCF-7) were obtained from the University of Tsukuba (Tsukuba, Ibaraki, Japan) [24].

2.2. Preparation of Soy Lecithin Liposomes. Liposomes were aseptically prepared by the thin-film hydration method. The lipid phase components (soy lecithin, cholesterol, CTAB, LET, and PTX) were accurately weighed and dissolved in chloroform:methanol mixture $(2: 1, v / v)$, in the ratio of 9:1 soy lecithin:cholesterol, $1 \%$ CTAB, 5\% LET, and 5\% PTX. The solution was transferred to a round-bottom flask and connected to a BUCHI rotavapor R-114 and BUCHI water bath B-480 with applied vacuum and temperature maintained at $45^{\circ} \mathrm{C}$ until the complete evaporation of solvents. The obtained dry, thin lipid film was hydrated with $0.5 \%$ Tween 80 by stirring at room temperature. The suspension was sonicated by the probe sonicator for $30 \mathrm{~min}$ and was further homogenized by a miniextruder (EmulsiFlex05 homogenizer, Avestin Inc., Ottawa, Canada) for 10 cycles. The obtained liposomal suspension was centrifuged at $16000 \mathrm{rpm}$ for $30 \mathrm{~min}$ to separate the unencapsulated drugs. The resulting formulation was lyophilized and stored at $2-8^{\circ} \mathrm{C}$ for further analysis.

2.3. Characterizations. The size distribution and zeta potential of the liposomal formulations were characterized by dynamic light scattering (DLS) using a Zetasizer Nano SZ (SZ-100, Horiba). The measurement was determined through a helium-neon $(\mathrm{He}-\mathrm{Ne})$ laser beam with the detection angle and the temperatures as $90^{\circ}$ and $25^{\circ} \mathrm{C}$, respectively. Samples were diluted with deionized water prior to measurement to reach the phospholipid concentration of $1000 \mathrm{ppm}$. The morphology of LET-PTX-Lips was examined by a transmission electron microscope (TEM) using JEM-1400, JEOL (Tokyo, Japan). The sample was diluted with deionized water $(1 \mathrm{mg} / \mathrm{mL})$. One drop of the liposomal formulation was deposited onto a carbon-copper grid (300-mesh, Ted Pella, Inc., USA) and air-dried at room temperature.

2.4. Stability Study. Lips, LET-Lips, and LET-PTX-Lips were preserved in a fridge at $2-8^{\circ} \mathrm{C}$ for one week and measured by their size and zeta value.

2.5. Determination of Encapsulation Efficiency and In Vitro Release Study. High Performance Liquid Chromatography (HPLC) was applied for the identification and quantitation of LET and PTX in the liposomal formulations using Flexar PDA Plus LC Detector (PerkinElmer, USA). The mobile phase consisted of acetonitrile/water with the volume ratio of $(40: 60)$. The mobile phase was degassed prior to use and 
delivered isocratic ally at a flow rate of $1 \mathrm{~mL} / \mathrm{min}$ through the reverse-phase Fortis C18 column $(150 \mathrm{~mm}, 4.6 \mathrm{~mm}$, pore size $5 \mu \mathrm{m}$; Fortis Technologies Ltd., Cheshire, UK), and a UV detector at $227 \mathrm{~nm}$ was used to monitor the column eluent. For determining the LET and PTX concentration in the liposomal formulations, a slight modification of the ultracentrifuge method of Yang et al. was applied [25]. An aliquot of $1 \mathrm{~mL}$ of the formulation was mixed with $10 \mathrm{~mL}$ of PBS $(\mathrm{pH} 7.4)$ and centrifuged at $1000 \mathrm{rpm}$ for $10 \mathrm{~min}$ at $25^{\circ} \mathrm{C}$. Then, centrifugation at $16000 \mathrm{rpm}$ for $30 \mathrm{~min}$ was performed to precipitate $11 \mathrm{~mL}$ of liposome supernatant, which was then decanted and washed twice with PBS (pH 7.4). The liposome pellets were then dissolved with $6 \mathrm{~mL}$ solvent and sonicated for $10 \mathrm{~min}$ for subsequent characterization. Collected samples were filtered through the $0.22 \mu \mathrm{m}$ PTFE syringe filters prior to analysis.

The encapsulation efficiency (EE\%) of the liposomal formulation for each drug was calculated using the following equation [26]:

$$
\operatorname{EE}(\%)=\frac{W_{p}}{W_{s}} \times 100
$$

where $W_{p}$ is the amount of drugs in the liposome pellet and $W_{s}$ is the amount of drugs in the liposome suspension.

The release profile of liposomal formulation was studied in vitro in PBS buffer ( $\mathrm{pH} 7.4)$ at the presence of $2 \%$ Tween 80. About $1 \mathrm{~mL}$ of the liposomal formulation was tightly sealed in a dialysis bag (MW cutoff $3.5 \mathrm{kDa}$ ) and immersed in $10 \mathrm{~mL}$ dialysis medium. The release study was performed at $37^{\circ} \mathrm{C}$ in a shaker bath $(100 \mathrm{rpm})$. At defined time intervals $(1,2,3,6,12,24,36,48,60$, and $72 \mathrm{~h})$, an aliquot of $1 \mathrm{~mL}$ sample was taken from the release medium followed by the immediate supplementation of the equal volume of the fresh medium. Controls containing free drug were prepared in the amount equal to the amount of the drug contained in the liposome and tested along with the liposomal dispersions. Samples were filtered (pore size $=0.22 \mu \mathrm{m}$ ) before being analysed by the above-mentioned HPLC method to determine the LET and PTX content.

2.6. Release Kinetics Study. To analyse the in vitro release patterns of both LET and PTX in coloaded form LETPTX-Lips, four drug release kinetic models, including zeroorder kinetic model, first-order kinetic order, Higuchi model, and Korsmeyer-Peppas model, were used. The zero-order kinetic model was the relationship between time and cumulative \% drug release, which could define the process of the constant drug released from a drug delivery system, and the drug level in the blood remained constant throughout the delivery. Meanwhile, the first-order kinetic model was the relationship of time and log cumulative \% of drug remaining. This model was applied to evaluate the concentrationdependent manner of the drug release. The Higuchi model was the relationship between the square root of time and cumulative $\%$ drug release, which was used to identify whether the prime mechanism of the drug release was a diffusion controlled release mechanism or not. Finally, the KorsmeyerPeppas model was the relationship of time and log cumulative
$\%$ drug release which helped to understand the dissolution mechanisms of the drugs from the matrix. Graphs of the zero-order, first-order, Higuchi, and Korsmeyer-Peppas models were drawn based on equations (2), (3), (4), and (5), respectively, and Microsoft Excel, then the rate constant and correlation values were obtained by applying a linear regression fit $[27,28]$.

$$
\begin{aligned}
C & =k_{0} t, \\
\log (100-C) & =-\frac{k_{\mathrm{f}} t}{2.303}, \\
\mathrm{C} & =\mathrm{k}_{\mathrm{H}} \sqrt{t}, \\
\mathrm{C} & =\mathrm{k}_{\mathrm{K}} \mathrm{t}^{\mathrm{n}},
\end{aligned}
$$

where $C$ is the cumulative $\%$ drug released at time $t, k_{0}$ is the zero-order rate constant, $k_{\mathrm{f}}$ is the first-order rate constant, $k_{\mathrm{H}}$ is the Higuchi dissolution constant, $k_{\mathrm{K}}$ is the KorsmeyerPeppas constant, and $n$ is the exponent that describes a particular diffusion mechanism.

2.7. Cell Culture and Viability Test. The cells were grown in DMEM/F-12 containing 10\% FBS and 2\% penicillin/streptomycin $(10,000 \mathrm{U} / \mathrm{mL}$ penicillin and $10 \mathrm{mg} / \mathrm{mL}$ streptomycin) in humidified air with $5 \% \mathrm{CO}_{2}$ at $37^{\circ} \mathrm{C}$. Cells were inoculated in a 96-well plate with a density of $3 \times 10^{4}$ cells/well. After $24 \mathrm{~h}$, cells were treated with reagents at a concentration of $10-100 \mu \mathrm{g} / \mathrm{mL}$ of Lips and $1 \mu \mathrm{g} / \mathrm{mL}$ of free LET, free LETPTX, LET-Lips, and LET-PTX-Lips for $24 \mathrm{~h}$, then $20 \mu \mathrm{L}$ of WST was added to each well and incubated for $4 \mathrm{~h}$ at $37^{\circ} \mathrm{C}$. Each plate was set to a microplate reader (IN Cell Analyzer $2500 \mathrm{HS}$ ), and the absorbance values were measured at $450 \mathrm{~nm}$. Results were expressed as cell viability (\%) by using equation (6). The cells, treated with medium, were considered as the control (100\% viable).

Cell viability $(\%)=\frac{\text { absorbance at } 450 \mathrm{~nm} \text { for sample }}{\text { absorbance at } 450 \mathrm{~nm} \text { for control }} \times 100 \%$.

2.8. Statistical Analysis. Quantitative data were expressed as mean \pm standard deviation for $n=3$. The statistical analysis was performed using ANOVA followed by Student's $t$-test with $p<0.05$ considered as statistically significant.

\section{Results and Discussion}

3.1. Characterizations of LET-PTX-Lips. The size and zeta potential of a nanocarrier play important roles to properly deliver drugs in the human body. Indeed, the best circulation time in the bloodstream can be obtained with particle size in between 100 and $200 \mathrm{~nm}$, which are small enough to go through the filtration of the spleen and selective uptake of the liver $[29,30]$. This size range allows nanoparticles to focus more efficiently on tumours. Meanwhile, the zeta potential is a good indicator to quantify the stability of nanoparticles in physiological conditions. It was reported that negative charges resulted in preventing fusion and 


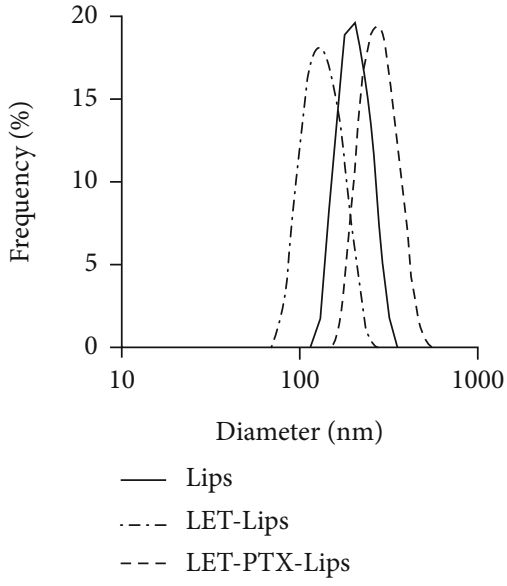

(a)

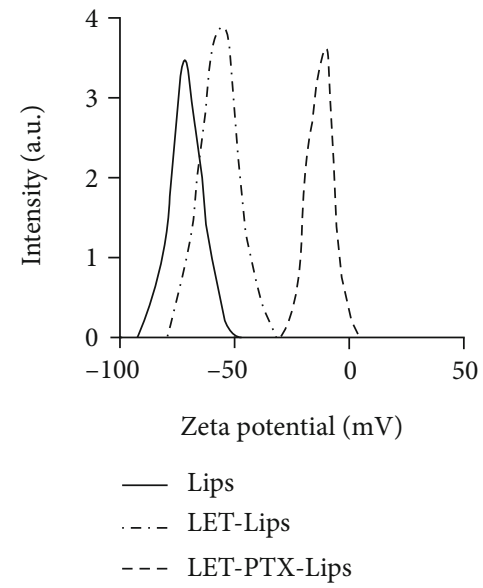

(b)

FIgure 1: (a) Size distribution and (b) zeta potential of Lips, LET-Lips, and LET-PTX-Lips.

aggregation and also reduced the phagocytosis [31, 32]. As exposed in Figure 1(a), the DLS particle sizes of Lips, LETLips, and the complex LET-PTX-Lips were $169.70 \pm 0.32$ $\mathrm{nm}, 216.20 \pm 2.47 \mathrm{~nm}$, and $193.10 \pm 8.70 \mathrm{~nm}$, respectively. The particle size of the obtained liposomes increased due to the increase of the hydrophobic drugs mainly residing in the lipid phase, and this phenomenon has also been observed in several previous studies. For instance, Sarfraz et al. showed an increase in liposome size from $127 \pm 11.14 \mathrm{~nm}$ with oleanolic acid loaded into lipid bilayer to $225.33 \pm 28.02 \mathrm{~nm}$ coencapsulated with doxorubicin [33]. Remarkably, the polydispersity index of all prepared samples was less than 0.5 , indicating that the size repartition is quite homogeneously distributed. Otherwise, as shown in Figure 1(b), the zeta potentials of Lips, LET-Lips, and LET-PTX-Lips were $69.20 \pm 0.55 \mathrm{mV},-54.50 \pm 0.89 \mathrm{mV}$, and $-13.75 \pm 4.41 \mathrm{mV}$, respectively. The drastic decrease in zeta potential of LETPTX-Lips $(-13.75 \pm 4.41 \mathrm{mV})$ was significantly lower than that of Lips $(-69.20 \pm 0.55 \mathrm{mV})$, which suggested that the stability of Lips has been reduced after codelivery of LET and PTX.

The TEM image showed that Lips and LET-PTX-Lips were spherical in terms of morphology, and the liposomal system possessed the size range which fell into the therapeutic-potential range (Figure 2). Moreover, no aggregation or fusion of Lips and LET-PTX-Lips was found, all of which were coherent with the DLS results. In light of these results, LET and PTX taken together might be encapsulated efficiently enough into Lips as spherical nanocarriers with an operational circulation in the human bloodstream.

3.2. Stability of the Synthesized Soy Lecithin Liposomes. The stability of Lips, LET-Lips, and LET-PTX-Lips was determined after a period of a week under $2-8^{\circ} \mathrm{C}$ conditions (Figure 3). During the studied period, all of Lips, LET-Lips, and LET-PTX-Lips presented similar effects with no significant change occurring in their size and zeta potential. The size of Lips increased to $172.70 \mathrm{~nm}$, while those values of LET-Lips and LET-PTX-Lips bounded to $225.10 \mathrm{~nm}$ and $201.50 \mathrm{~nm}$, respectively, on the last day of storage (Figure 3(a)). Similar trends happened on the zeta potential (Figure 3(b)) that the absolute value was decreased slowly and yet still remained negative after one week for all the samples $(-67.43 \mathrm{mV}$, $-50.21 \mathrm{mV}$, and $-12.22 \mathrm{mV}$ for Lips, LET-Lips and LET-PTXLips, respectively). The stability of these liposomal formulations was due to the constraint of the lipid hydrolysis rate existing in the lipid bilayer at cold temperatures $\left(2-8^{\circ} \mathrm{C}\right)$. Moreover, at temperatures below $35^{\circ} \mathrm{C}$, the lipid was in the gel phase which maintained their molecular conformation as well as the geometry of the lipid bilayer [14]. Therefore, Lips, LET-Lips, and LET-PTX-Lips were proven to be stable under the storing temperatures.

3.3. Drug Loading Efficiency and In Vitro Release Profiles. It should be noted that when using lipid nanoparticles as drug carriers, it is vital to determine and understand the drug that can be loaded to the nanoparticles. In this study, EE of LETPTX-Lips was determined to be $31.13 \pm 0.60 \%$ and $50.56 \pm$ $1.91 \%$ for LET and PTX, respectively. The difference between EE values of LET and PTX in Lips might be caused by their molecular size and weight. PTX with the significantly higher size and molecular weight could compete against LET and occupy more space inside the lipid bilayer during synthesis of LET-PTX-Lips. Besides, the lipophilic nature of the two drugs lead to competition between them for the hydrophobic space in the lipid bilayers during entrapment, as a similar case described in Shavi et al. and Deniz et al., where higher cholesterol concentrations lead to a decrease in the encapsulation efficiency of ANA and celecoxib [34, 35]. Through these results, EE of LET and PTX demonstrated the possibility of entrapping two drugs in the synthesized Lips with an important percentage of encapsulated drugs.

As shown in Figure 4, the in vitro drug release was conducted to analyse the behavior of LET, PTX, and LET-PTX and the capacity of Lips to control drug release. From the evaluation of results demonstrated in Figure 4, it can be established that LET and PTX exhibited similar release profiles. The fast release of free LET and free PTX was observed in $72 \mathrm{~h}$ (LET, $90.05 \% \pm 4.6 \%$; PTX, 94.79\% $\pm 4.5 \%$ ) while the late slow release is ascribed to the sustained release of the two 


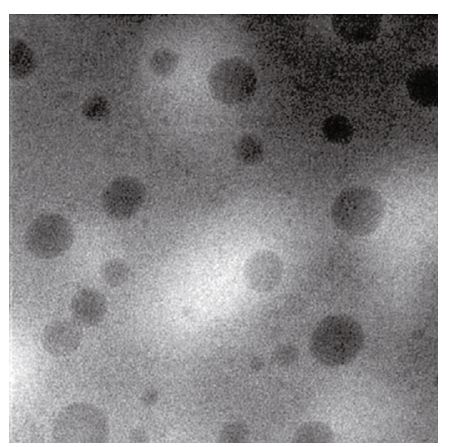

(a)

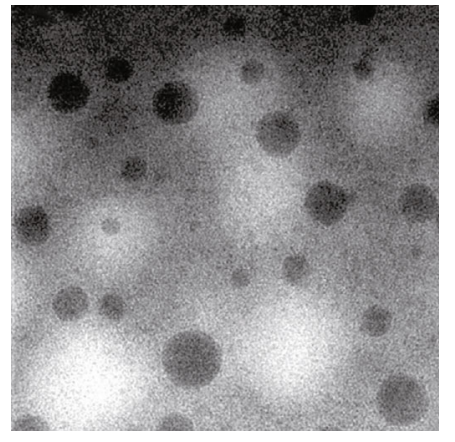

(c)

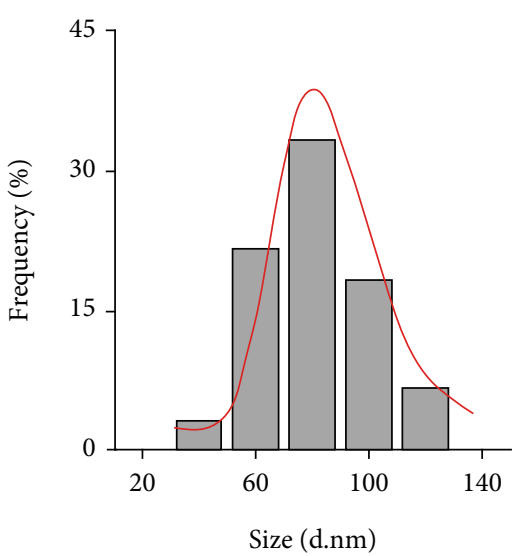

(b)

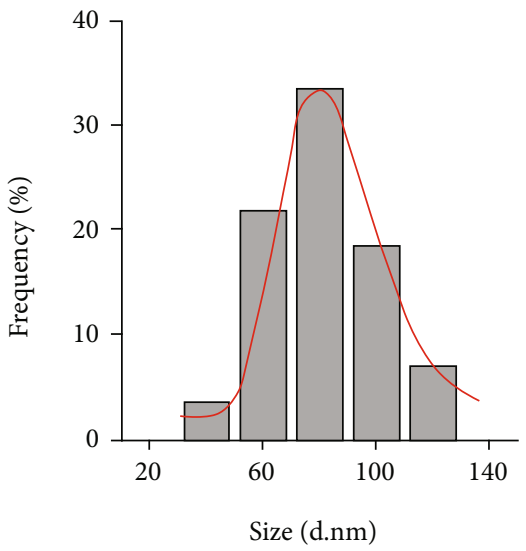

(d)

FIgUre 2: (a, c) TEM images at scale bar $200 \mathrm{~nm}$ and (b, d) particle size distribution of Lips and LET-PTX-Lips.

drugs from Lips. The cumulative release reached $31.70 \% \pm$ $3.2 \%$ for LET and $53.29 \% \pm 3.9 \%$ for PTX from Lips at $72 \mathrm{~h}$. In other words, the release behavior of drugs from loaded Lips was significantly slower than that of free drugs. This was similar to the previous studies, which indicated that the prepared Lips was shown to have a sustained release profile which was consistent with most liposomal drug delivery systems [36-38].

3.4. Kinetic Models of Drug Release. The release patterns of LET and PTX from LET-PTX-Lips in Figure 4 were fitted to the zero-order model, first-order model, Higuchi model, and Korsmeyer-Peppas model to determine the highest correlation with experimental results. The in vitro release study was conducted in $72 \mathrm{~h}$ and showed about $31 \%$ and $53 \%$ of the initial LET and PTX, respectively, were released. It was reported that the first $60 \%$ of drug release was typically sufficient for determining the best fit model of the drug release [39]. Therefore, the release data of the system in $72 \mathrm{~h}$ was used to fit to the four models. Table 1 lists the kinetic release parameters and regression coefficients calculated from the four kinetic models.

As seen in Table 1 and Figure 5, it was found that the Higuchi and Korsmeyer-Peppas models showed a higher degree of correlation coefficients $\left(R^{2}\right)$ than the two other models. Therefore, the discussion of LET-PTX-Lips release kinetics was focused on the Higuchi and Korsmeyer-Peppas models. LET was released followed the equation $y=3.2513$ $x+7.287\left(R^{2}=0.8355\right)$; meanwhile, PTX was released following the equation $y=5.8797 x+6.7726 \quad\left(R^{2}=0.9532\right)$. These results suggested that LET and PTX released from LET-PTX-Lips followed diffusion mechanism [40]. This is reasonable because the Higuchi model describes the release of active agents that are less solubly dispersed in homogeneous matrices and submitted to a diffusing medium with the consideration of the dissolution of a lipophilic, homogeneous, and planar matrix [41]. Moreover, the KorsmeyerPeppas model, which was developed based on the Higuchi model, is important to classify the possible release profile of active agents in dosage forms [42]. The model KorsmeyerPeppas power law equation stated the type of diffusion based on the slope values. Both the slope values of LET and PTX in Korsmeyer-Peppas equations $(n)$ were lower than 0.5 which implies that the two drugs released from LET-PTX-Lips may be modelled similar to a polymeric system undergoing degradation $[43,44]$.

3.5. Cytotoxicity Assay. The cytotoxicity of the samples was tested on MCF-7 cells via WST assay (Figure 6). For the WST test of Lips, the cells were noticed to have entirely 


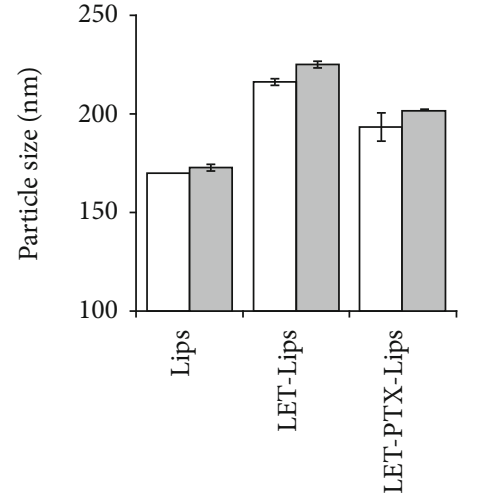

(a)

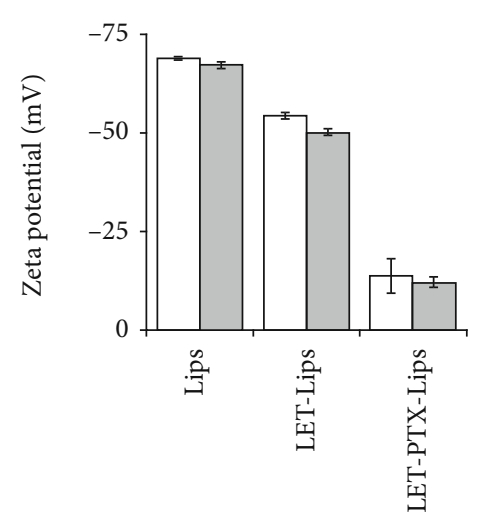

(b)

Figure 3: (a) Particle size distribution and (b) zeta potential of Lips, LET-Lips, and LET-PTX-Lips after preparation (blank column) and one week of storage at $2-8^{\circ} \mathrm{C}$ (gray column).

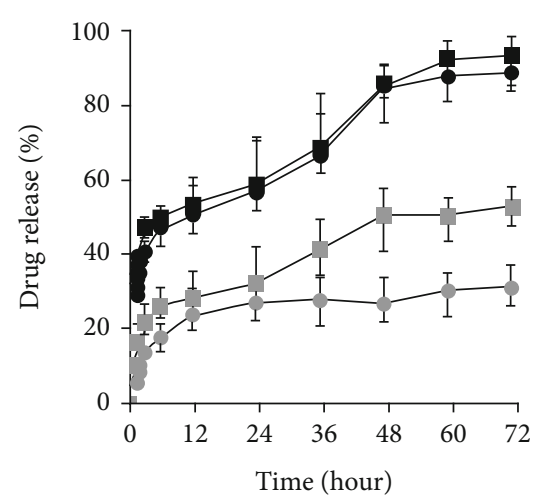

FIGURE 4: In vitro release profiles of free LET (black circle), free PTX (black square), and LET (grey circle) and PTX (grey square) from LET-PTX-Lips at $37^{\circ} \mathrm{C}$ in PBS $(\mathrm{pH}=7.4 ; n=3$, mean \pm standard deviation).

TABLE 1: Rate constants and correlation coefficients of LET-PTXLips obtained through the zero-order kinetic model, first-order kinetic model, Higuchi model, and Korsmeyer-Peppas model.

\begin{tabular}{lccc}
\hline Models & & LET & PTX \\
\hline \multirow{2}{*}{ Zero-order } & $k_{0}$ & 0.3247 & 0.6248 \\
& $R^{2}$ & 0.6524 & 0.8431 \\
First-order & $k_{\mathrm{f}}$ & 0.0018 & 0.0041 \\
& $R^{2}$ & 0.6941 & 0.9047 \\
Higuchi & $k_{\mathrm{H}}$ & 3.2513 & 5.8797 \\
\multirow{2}{*}{ Korsmeyer-Peppas } & $R^{2}$ & 0.8355 & 0.9532 \\
& $n$ & 0.3116 & 0.3502 \\
& $R^{2}$ & 0.8103 & 0.9495 \\
\hline
\end{tabular}

normal morphology until the highest concentration of Lips $(100 \mu \mathrm{g} / \mathrm{mL})$. Lips showed over $80 \%$ cell viability with concentrations ranging from $10 \mu \mathrm{g} / \mathrm{mL}$ to $100 \mu \mathrm{g} / \mathrm{mL}$, which indicated a potential nanocarrier with great biocompatibility in cancer treatment (Figure 6(b)). Also, the result of the WST assay of Lips demonstrated that the cytotoxic effect seen with drug-loading Lips was solely due to the drugs which were released from the system. On the other hand, LET and LET-PTX in free forms as well as LET and LET-PTX loaded in Lips at the concentration of $1 \mu \mathrm{g} / \mathrm{mL}$ of LET were treated on MCF-7 cells to evaluate the contribution of PTX to the anticancer efficacy of LET. Most cells were not damaged when exposed to free LET; meanwhile, at the same concentration of LET, free LET-PTX did not show the intact shape of cells (Figure 6(a)). Investigated under a light microscope with 20x objective lens, cells were either in the pyknosis state, in which the nucleus shrunk and the cells' content was condensed at the center or periphery, or in the karyorrhexis state, in which the nucleus was disintegrated (data not shown), indicating the progress of the intrinsic pathway of apoptosis. A similar trend occurred when comparing the cell viability between LET-Lips and LET-PTX-Lips samples: LET-Lips gave about $85 \%$ of surviving cells versus LET-PTX-Lips which gave about $78 \%$ of surviving cells (Figure $6(\mathrm{~b})$ ). These results showed that PTX could contribute to the increased efficacy of LET in both free forms and liposome-loaded forms. It can be explained that the free drug was uptaken by the cell via passive diffusion of a higher concentration gradient, causing the immediate toxicity in cells [45], whilst the drug loaded into the liposome was taken up via receptormediated endocytosis, by which the liposome vesicle fuses with the lysosome and ends up being digested. Additionally, it could be observed that the Lips coencapsulation of LET and PTX showed an obviously higher cell viability than the drugs in free forms, which was explained by the slow release that had been shown in an in vitro drug release study, indicating that Lips potentiated the capability of controlling drug release.

\section{Conclusions}

The formulation of drug delivery systems composed of Lips encapsulating LET and PTX has been working fine using the thin film hydration method. The resulting sample shows in TEM images the spherical particle with a diameter around $193 \mathrm{~nm}$, staying in the required range of $100-200 \mathrm{~nm}$. The 


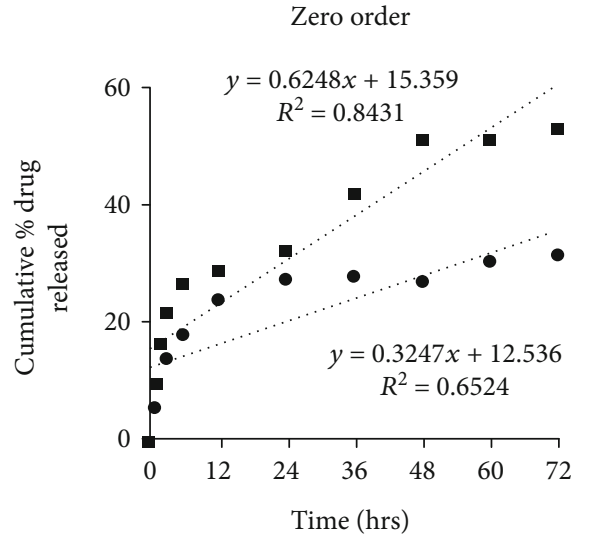

(a)

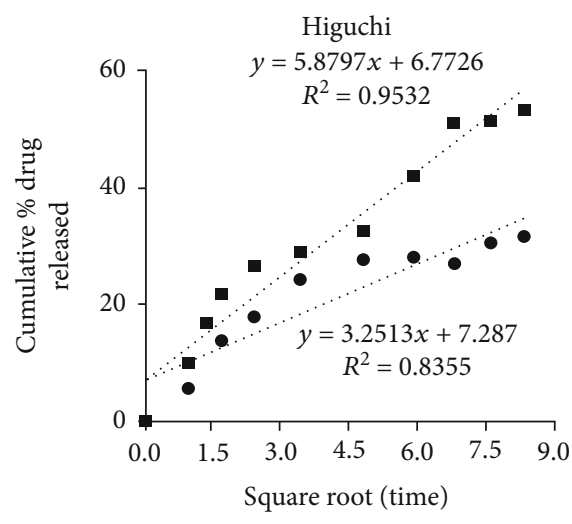

(c)

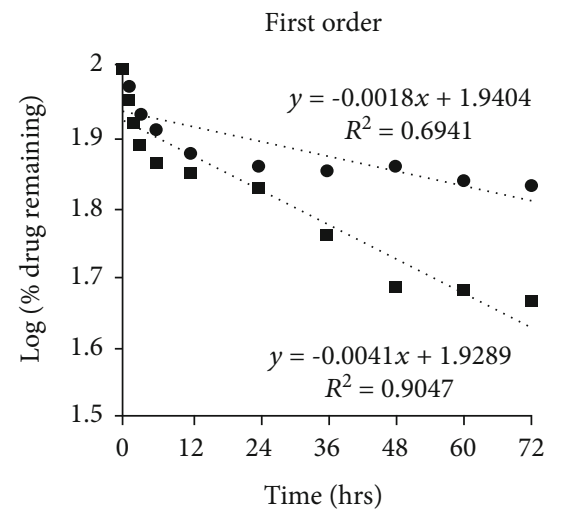

(b)

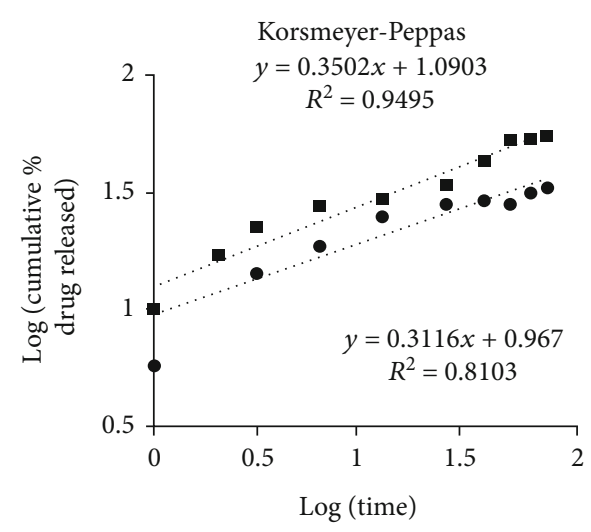

(d)

FIgure 5: Release kinetics of Letrozole (solid circle) and Paclitaxel (solid square) from LET-PTX-Lips fitted to four kinetic models: (a) zeroorder kinetic model, (b) first-order kinetic model, (c) Higuchi model, and (d) Korsmeyer-Peppas model.
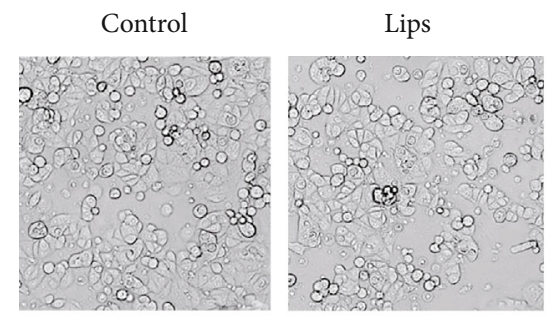

A

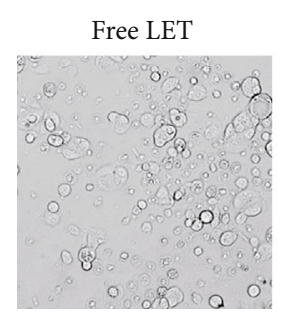

Free PTX

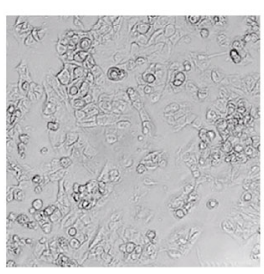

B

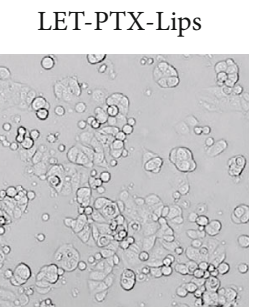

Free LET-PTX

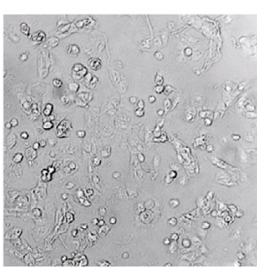

(a)
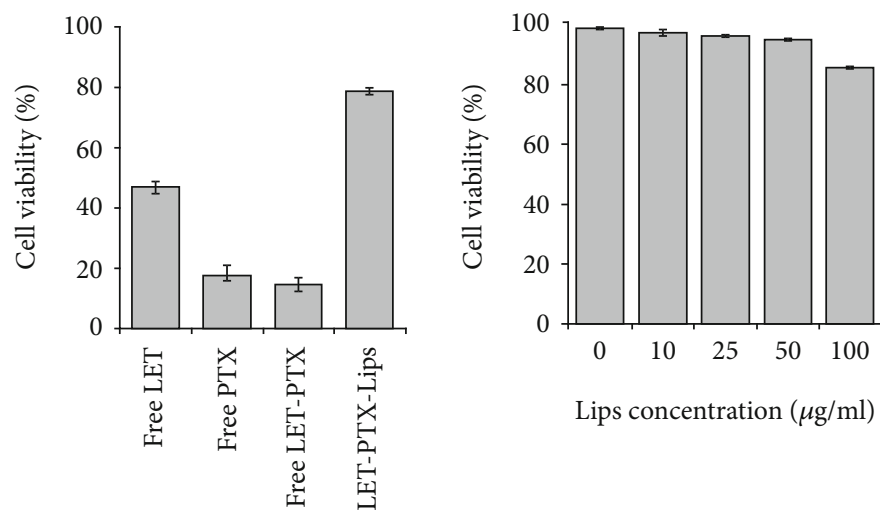

Lips concentration $(\mu \mathrm{g} / \mathrm{ml})$

A

(b)

FIGURE 6: (a) Images of MCF-7 cells incubated with (A) control, free LET, and free LET-PTX at a concentration of $1 \mu \mathrm{g} / \mathrm{mL}$ of LET and (B) Lips $(100 \mu \mathrm{g} / \mathrm{mL})$, LET-Lips, and LET-PTX-Lips at a concentration of $1 \mu \mathrm{g} / \mathrm{mL}$ of LET (scale bar $=100 \mathrm{~nm}$ ) for $24 \mathrm{~h}$. (b) Viability of MCF-7 cells incubated with (A) free LET, free LET-PTX, LET-Lips, and LET-PTX-Lips at a concentration of $1 \mu \mathrm{g} / \mathrm{mL}$ of LET and (B) Lips at different concentrations for $24 \mathrm{~h}$. The data represent the mean values \pm the standard deviation (SD) $(n=4)$. 
zeta potential results show particles negatively charged making the LET-PTX-Lips complex a potential candidate for in vivo drug release. HPLC results reveal EE of $31.13 \pm$ $0.60 \%$ and $50.56 \pm 1.91 \%$ for LET and PTX, respectively. Moreover, the release profiles which followed the Higuchi model prove the prolonged release of LET and PTX. The WST assay indicated an obvious increase of the toxicity of the complex compared to the single drug. After all these measurements and results, it seems that the prepared LET-PTXLips could be a potential drug delivery system with the goal of treating cancer.

\section{Data Availability}

The data used to support the findings of this study are included within the article.

\section{Conflicts of Interest}

The authors declare that there is no conflict of interest regarding the publication of this paper.

\section{Acknowledgments}

This work was supported by the Vietnam National Foundation for Science and Technology Development (NAFOSTED) under grant number 104.06-2018.320.

\section{References}

[1] D. X. He and X. Ma, "Clinical utility of letrozole in the treatment of breast cancer: a Chinese perspective," Oncotargets and Therapy, vol. 9, pp. 1077-1084, 2016.

[2] A. Yassemi, S. Kashanian, and H. Zhaleh, "Folic acid receptortargeted solid lipid nanoparticles to enhance cytotoxicity of letrozole through induction of caspase-3 dependentapoptosis for breast cancer treatment," Pharmaceutical Development and Technology, vol. 25, no. 4, pp. 397-407, 2020.

[3] B. Alemrayat, A. Elhissi, and H. M. Younes, "Preparation and characterization of letrozole-loaded poly (d, l-lactide) nanoparticles for drug delivery in breast cancer therapy," Pharmaceutical Development and Technology, vol. 24, no. 2, pp. 235242, 2019.

[4] S. Johnston, M. Pegram, M. Press et al., Lapatinib combined with letrozole vs. letrozole alone for front line postmenopausal hormone receptor positive (HR+) metastatic breast cancer (MBC): first results from the EGF30008 Trial, AACR, 2009.

[5] L. S. Schwartzberg, S. X. Franco, A. Florance, L. O'Rourke, J. Maltzman, and S. Johnston, "Lapatinib plus Letrozole as First-Line Therapy for HER-2+Hormone Receptor-Positive Metastatic Breast Cancer," The oncologist, vol. 15, no. 2, pp. 122-129, 2010.

[6] A. C. Wolff, A. A. Lazar, I. Bondarenko et al., "Randomized phase III placebo-controlled trial of letrozole plus oral temsirolimus as first-line endocrine therapy in postmenopausal women with locally advanced or metastatic breast cancer," Journal of clinical oncology, vol. 31, no. 2, pp. 195-202, 2013.

[7] R. S. Finn, J. P. Crown, I. Lang et al., "The cyclin-dependent kinase 4/6 inhibitor palbociclib in combination with letrozole versus letrozole alone as first-line treatment of oestrogen receptor-positive, HER2-negative, advanced breast cancer
(PALOMA-1/TRIO-18): a randomised phase 2 study," The Lancet Oncology, vol. 16, no. 1, pp. 25-35, 2015.

[8] F. A. Fisusi and E. O. Akala, "Drug combinations in breast cancer therapy," Pharmaceutical nanotechnology, vol. 7, no. 1, pp. 3-23, 2019.

[9] D. Chen, W. Hackl, O. Ortmann, and O. Treeck, "Effects of a combination of exemestane and paclitaxel on human tumor cells in vitro," Anti-Cancer Drugs, vol. 15, no. 1, pp. 55-61, 2004.

[10] O. C. Olson, H. Kim, D. F. Quail, E. A. Foley, and J. A. Joyce, "Tumor-associated macrophages suppress the cytotoxic activity of antimitotic agents," Cell Reports, vol. 19, no. 1, pp. 101113, 2017.

[11] S. R. Hall, The Anticancer Activity and Mechanisms of Action of Jadomycins in Multidrug Resistant Human Breast Cancer Cells, Dalhousie Univeristy, 2018.

[12] K. Samanta, S. Setua, S. Kumari, M. Jaggi, M. M. Yallapu, and S. C. Chauhan, "Gemcitabine combination nano therapies for pancreatic cancer," Pharmaceutics, vol. 11, no. 11, p. 574, 2019.

[13] P. Schöffski, S. Cresta, I. A. Mayer et al., "A phase Ib study of pictilisib (GDC-0941) in combination with paclitaxel, with and without bevacizumab or trastuzumab, and with letrozole in advanced breast cancer," Breast Cancer Research, vol. 20, no. 1, p. 109, 2018.

[14] N. T. T. Le, V. D. Cao, T. N. Q. Nguyen, T. T. H. Le, T. T. Tran, and T. T. Hoang Thi, "Soy lecithin-derived liposomal delivery systems: surface modification and current applications," International Journal of Molecular Sciences, vol. 20, no. 19, pp. 127, 2019.

[15] U. Bulbake, S. Doppalapudi, N. Kommineni, and W. Khan, "Liposomal formulations in clinical use: an updated review," Pharmaceutics, vol. 9, no. 4, p. 12, 2017.

[16] H. He, Y. Lu, J. Qi, Q. Zhu, Z. Chen, and W. Wu, “Adapting liposomes for oral drug delivery," Acta Pharmaceutica Sinica $B$, vol. 9, no. 1, pp. 36-48, 2019.

[17] M. Li, C. Du, N. Guo et al., "Composition design and medical application of liposomes," European Journal of Medicinal Chemistry, vol. 164, pp. 640-653, 2019.

[18] N. T. T. Le, D. T. D. Nguyen, N. H. Nguyen, C. K. Nguyen, and D. H. Nguyen, "Methoxy polyethylene glycol-cholesterol modified soy lecithin liposomes for poorly water-soluble anticancer drug delivery," Journal of Applied Polymer Science, vol. 138, p. 49858, 2020.

[19] H. Daraee, A. Etemadi, M. Kouhi, S. Alimirzalu, and A. Akbarzadeh, "Application of liposomes in medicine and drug delivery," Artificial cells, nanomedicine, and biotechnology, vol. 44, no. 1, pp. 381-391, 2014.

[20] M. Maniyar, A. Chakraborty, and C. Kokare, "Formulation and evaluation of letrozole-loaded spray dried liposomes with PEs for topical application," Journal of Liposome Research, vol. 30, no. 3, pp. 274-284, 2019.

[21] A. Zajdel, A. Wilczok, K. Jelonek et al., "Cytotoxic effect of paclitaxel and lapatinib co-delivered in polylactide-co-poly (ethylene glycol) micelles on HER-2-negative breast cancer cells," Pharmaceutics, vol. 11, no. 4, p. 169, 2019.

[22] M. S. Franco, M. C. Roque, and M. C. Oliveira, "Short and long-term effects of the exposure of breast cancer cell lines to different ratios of free or co-encapsulated liposomal paclitaxel and doxorubicin," Pharmaceutics, vol. 11, no. 4, p. 178, 2019.

[23] T. L. Nguyen, T. H. Nguyen, C. K. Nguyen, and D. H. Nguyen, "Redox and $\mathrm{pH}$ responsive poly (amidoamine) dendrimer- 
heparin conjugates via disulfide linkages for letrozole delivery," BioMed Research International, vol. 2017, 7 pages, 2017.

[24] B. Shashni and Y. Nagasaki, "Nitroxide radical-containing nanoparticles attenuate tumorigenic potential of triple negative breast cancer," Biomaterials, vol. 178, pp. 48-62, 2018.

[25] T. Yang, F.-D. Cui, M.-K. Choi et al., "Liposome formulation of paclitaxel with enhanced solubility and stability," Drug Delivery, vol. 14, no. 5, pp. 301-308, 2008.

[26] T. L. Nguyen, T. H. Nguyen, and D. H. Nguyen, "Development and in vitro evaluation of liposomes using soy lecithin to encapsulate paclitaxel," International Journal of Biomaterials, vol. 2017, 7 pages, 2017.

[27] M. Barzegar-Jalali, K. Adibkia, H. Valizadeh et al., "Kinetic analysis of drug release from nanoparticles," Journal of Pharmacy \& Pharmaceutical Sciences, vol. 11, no. 1, pp. 167-177, 2008.

[28] C. G. England, M. C. Miller, A. Kuttan, J. O. Trent, and H. B. Frieboes, "Release kinetics of paclitaxel and cisplatin from two and three layered gold nanoparticles," European Journal of Pharmaceutics and Biopharmaceutics, vol. 92, pp. 120-129, 2015.

[29] E. Nance and M. McKenna, "Challenges and barriers," in Nanoparticles for Biomedical Applications, pp. 89-107, Elsevier, 2020.

[30] N. T. T. Le, L. P. T. Pham, D. H. T. Nguyen et al., "Liposomebased nanocarrier system for phytoconstituents," Novel Drug Delivery Systems for Phytoconstituents, p. 45, 2019.

[31] N. A. Charoo, Z. Rahman, and M. A. Khan, "Nanoparticles for improvement in oral bioavailability," in Nanoarchitectonics in Biomedicine, pp. 371-410, Elsevier, 2019.

[32] D. H. Surve, P. Dandekar, P. V. Devarajan, and A. B. Jindal, "Intracellular delivery: an overview," in Targeted Intracellular Drug Delivery by Receptor Mediated Endocytosis, pp. 3-41, Springer, 2019.

[33] M. Sarfraz, A. Afzal, T. Yang et al., "Development of dual drug loaded nanosized liposomal formulation by a reengineered ethanolic injection method and its pre-clinical pharmacokinetic studies," Pharmaceutics, vol. 10, no. 3, p. 151, 2018.

[34] G. V. Shavi, M. S. Reddy, R. Raghavendra et al., "PEGylated liposomes of anastrozole for long-term treatment of breast cancer: in vitro and in vivo evaluation," Journal of Liposome Research, vol. 26, no. 1, pp. 28-46, 2015.

[35] A. Deniz, A. Sade, F. Severcan, D. Keskin, A. Tezcaner, and S. Banerjee, "Celecoxib-loaded liposomes: effect of cholesterol on encapsulation and in vitro release characteristics," Bioscience Reports, vol. 30, no. 5, pp. 365-373, 2010.

[36] K. Jiang, M. Shen, and W. Xu, “Arginine, glycine, aspartic acid peptide-modified paclitaxel and curcumin co-loaded liposome for the treatment of lung cancer: in vitro/vivo evaluation," International Journal of Nanomedicine, vol. 13, pp. 25612569, 2018.

[37] J. Meng, F. Guo, H. Xu, W. Liang, C. Wang, and X.-D. Yang, "Combination Therapy using Co-encapsulated Resveratrol and Paclitaxel in Liposomes for Drug Resistance Reversal in Breast Cancer Cells _in vivo_,"Scientific Reports, vol. 6, no. 1, p. 22390, 2016

[38] L. R. Tefas, B. Sylvester, I. Tomuta et al., "Development of antiproliferative long-circulating liposomes co-encapsulating doxorubicin and curcumin, through the use of a quality-bydesign approach," Drug design, development and therapy, vol. 11, pp. 1605-1621, 2017.
[39] R. Bettini, P. L. Catellani, P. Santi, G. Massimo, N. A. Peppas, and P. Colombo, "Translocation of drug particles in HPMC matrix gel layer: effect of drug solubility and influence on release rate," Journal of Controlled Release, vol. 70, no. 3, pp. 383-391, 2001.

[40] C. Subal, Modelling of Drug Release: The Higuchi Equation and its Application, 2006, Pharmabiz. com.

[41] M. L. Bruschi, "Mathematical models of drug release," Strategies to modify the drug release from pharmaceutical systems, 2015.

[42] N. A. Peppas and B. Narasimhan, "Mathematical models in drug delivery: how modeling has shaped the way we design new drug delivery systems," Journal of Controlled Release, vol. 190, pp. 75-81, 2014.

[43] J. Siepmann and N. A. Peppas, "Modeling of drug release from delivery systems based on hydroxypropyl methylcellulose (HPMC)," Advanced drug delivery reviews, vol. 64, pp. 163174, 2012.

[44] G. Singhvi and M. Singh, "In-vitro drug release characterization models," International Journal of Pharmaceutical Studies and Research, vol. 2, no. 1, pp. 77-84, 2011.

[45] J. Sun, L. Jiang, Y. Lin et al., "Enhanced anticancer efficacy of paclitaxel through multistage tumor-targeting liposomes modified with RGD and KLA peptides," International Journal of Nanomedicine, vol. 12, pp. 1517-1537, 2017. 